\title{
Fragwürdige Entwicklung
}

Rolf Adler

Prof. em. Dr. med., Mitglied FMH

\author{
Eine Diagnoseliste bildet von nun an im Kanton Luzern, und vielleicht bald in der \\ ganzen Schweiz, die Entscheidungsgrundlage, welche Erkrankungen stationär oder \\ ambulant behandelt werden sollen. Somit wird der Mensch auf eine Diagnose \\ reduziert. Zeit, die Diagnoselisten aus wissenschaftlicher, praktischer und finan- \\ zieller Sicht zu hinterfragen.
}

Das Vorgehen weckt Erinnerungen an eine Bildergeschichte von Giovanetti, welche vor vielen Jahren im Nebelspalter erschien. Im ersten Bild steht Max, Giovanettis Murmeltier-Maskottchen, vor einem Tannenbaum, die linke Hand zum Schutz in einem Boxhandschuh, die rechte ein Beil haltend. In folgenden Bild sieht man Max, der zuerst mit seinem Beil nur an der Baumspitze einige Zweige abhackt. Im dritten Bild hat er schon einige grössere Äste entfernt. So geht es weiter, bis schlussendlich nur noch ein astloses Stämmchen in die Höhe ragt. Max steckt derweil hüfthoch in Asthaufen. Gehen alle Maxe im Kanton Luzern und später in der ganzen Schweiz gleichermassen vor, so gibt es bald nur noch kahle, uniforme Stämme. Doch was hat dieses Cartoon nun mit der Thematik der Diagnoselisten zu tun?

\section{Grundlage unserer Diagnosen}

Im Zentrum steht doch die Definition des Begriffs «Diagnose». Eine solche kommt zustande, indem wir Veränderungen in unserer Umwelt, beispielsweise am menschlichen Körper, zu Mustern zusammenstellen. Daraus leiten wir eine Anweisung für das weitere Vorgehen ab. Die Diagnose ist, wie Uexküll und Wesiack es bezeichnet haben, eine Handlungsanweisung. So hiess die Vitamin- $B_{12}$-Mangel-Anämie einst "Perniziosa», weil sie unbehandelbar war. Die Handlungsanweisung lautete demnach: "Da ist nichts mehr zu machen.» Heute wird die Vitamin- $B_{12}$-Mangel-Anämie als solche bezeichnet, da es zwischenzeitlich entsprechende Vitaminpräparate gibt.

Diagnosen basieren auf medizinischen Theorien, die wiederum ein Produkt unserer Ausbildung zum Arzt sind. Diagnosen sind ein von uns gemachtes Konstrukt und nicht «gegeben». Das derzeit vorherrschende theoretische Konzept der Medizin ist stark mathematisch- physikalisch-chemisch geprägt. Es hat zu grossen Fortschritten in der Medizin geführt, aber auch zu einer Medizin mit Körpern ohne Seelen und einer Medizin mit Seelen ohne Körper.

\section{Den Patienten, nicht nur die Krankheit behandeln}

Unsere Ausbildung vermittelt vor allem sogenannte Fakten. Wissenschaftstheoretische Inhalte wie beispielsweise Medizinkonzepte, die unsere ärztliche Entscheidungsfindung beeinflussen, werden nicht besprochen. In der Folge sind sich die Mediziner nicht bewusst, dass sie ihre Welt nach einer Theorie konstruieren. Den nackten, übrig gebliebenen Stamm des Tannenbaums in Giovanettis Zeichnung vergleiche ich hier mit der mathematisch-physikalisch-chemischen Medizin, die Zweige und Nadeln mit den psychosozialen Aspekten. Der Tannenbaum als lebendiges Wesen existiert nicht mehr.

Auf den Menschen bzw. Patienten bezogen gilt das Gleiche. Unsere Wissenschaft ist die Wissenschaft von der Natur des Menschen - und diese ist eine bio-psycho-soziale. Psycho-sozial heisst, dass die Lebensumstände und die Geschichte des Menschen eine Rolle in Gesundheit und Krankheit spielen. Ein Beispiel: Eine schwere Kindheit führt unter anderem durch Methylierung zu Veränderungen am Erbgut. In der Folge kommt es zu Abschaltungen von Genen und damit zu Veränderungen am Aufbau des Organismus. Belastete Kinder erkranken im Erwachsenenalter häufiger als unbelastete Kinder an Krankheiten, die vom Psychischen bis zum Körperlichen reichen. C. Thurston et al. [1] wiesen kürzlich nach, dass misshandelte Mädchen später als Frauen mittleren Alters vermehrt subklinische kardiovaskuläre Störungen aufweisen als nichtmisshandelte Mädchen. 
Der Neurochirurg Harvey Cushing (1869 bis 1939), der übrigens 1900 als Assistent bei Theodor Kocher in Bern arbeitete, prägte den Satz: «Der Arzt hat nicht nur die Krankheit zu betrachten, sondern den Menschen mit seiner Krankheit, und nicht nur den Menschen mit seiner Krankheit, sondern den Menschen mit seiner Krankheit in seiner Umwelt.» Francis Peabody (1881 bis 1927) drückte sich ähnlich aus: «The secret of the care of the patient is in caring for the patient.» William Osler (1849 bis 1919) zum gleichen Thema: "The good physician treats the disease, the great physician treats the patient who has the disease.»

\section{Beispiel aus dem Alltag}

Wenn nur biomedizinisch, also nach Diagnosis Related Groups und Diagnoselisten vorgegangen wird, sind die Folgen verheerend, wie folgendes Beispiel zeigt: Ein älteres Emigrantenpaar aus dem Balkan, das schon viele Jahre in Bern lebt, wohnt im dritten Stock eines Miethauses ohne Lift. Die Frau ist invalid, sitzt tagsüber fast nur zeitungslesend im Lehnstuhl. Der Mann umsorgt sie, kocht, wäscht, putzt, wechselt die Bettwäsche und kauft ein. Er leidet an einer Coxarthrose und muss operiert werden, nachdem Analgetika und Physiotherapie seine Schmerzen nicht mehr lindern. Er wird erfolgreich operiert, das biomedizinische Modell war erfolgreich. Er wird bereits am vierten Tag nach Hause entlassen. Weder ist der Stellvertreter des Hausarztes noch die Spitex über den Patienten orientiert. Zudem ist der zugewiesene Physiotherapeut ausgebucht. Die im Parterre wohnhafte, von einer Invalidenrente lebende, frühere Kinderpflegefachfrau beobachtet, wie der Mann seine Aufgaben kaum mehr bewältigen kann. Sie greift ein und vermeidet so eine Katastrophe.

\section{DRG und Diagnosegruppen sind Zeichen für einen Missstand}

Obwohl Generationen von Ärztinnen und Ärzten Peabodys Haltung kennen, wird sie unter dem Druck der Politiker, Krankenkassenagenten und Gesundheitsökonomen nicht mehr beherzigt. Das angeführte Beispiel, die Diagnosis Related Groups und Diagnoselisten sind Belege dafür. Eine Untersuchung von Dr. Doris Brühlmeier in der SÄZ [2] zeigt drastisch, dass die Gutachterinnen und Gutachter der Invalidenversicherung ebenfalls nach dem biomedizinischen Konzept vorgehen und menschliche Katastrophen auslösen. Die Autorin und praktizierende Psychiaterin gelangte in den letzten Jahren zur Überzeugung, dass die systematisch «härtere Gangart» der Invalidenversicherung bei psychisch-psychosomatisch Kranken und bei Schmerzpatienten kontraproduktiv ist. Frau Brühlmeiers Nachzählung erhärtete den Verdacht. Zu Unrecht abgewiesene Kranke und Teilberentete, denen ungerechtfertigterweise die Rente entzogen wird, geraten in existentielle Krisen. Während Teilberentete, die ihre Teilrente behalten, in den meisten Fällen arbeiten.

In der SÄZ fordern die Herren Mast und Schneuwly [3] in ihrem Artikel «Mehr Verständnis für die eidgenössische Versicherungsmedizin» und führen an: «Ein weiteres Missverständnis betrifft den sozialen Kontext der Versicherten. Die Regularien zu versicherungsmedizinischen Leistungen schliessen die Berücksichtigung von sozialen Kontextfaktoren (schwierige Familienverhältnisse, konflikthafte Partnerschaften, finanzielle Probleme, kultureller und ethnischer Hintergrund u.a.m.) aus. Hier handelt es sich also nicht um eine Ignoranz der Gutachter, sondern um eine Vorgabe des Gesetzgebers bzw. der Kostenträger.»

Die selbstattestierte Nicht-Ignoranz von Mast und Scheuwly vermag nicht überzeugen. Mast ignoriert, dass sein Wissenschaftskonzept aus dem 17. Jahrhundert längst überholt ist. Und von Schneuwly können wir nicht erwarten, dass er von Medizin etwas versteht. Leider, und dies verdient ein Ausrufezeichen, haben die beiden Autoren das menschliche Elend übersehen. Das ist meiner Ansicht nach unverzeihlich.

\section{Vernichtende Schlussfolgerungen}

Welche Schlussfolgerungen lassen sich nun ziehen? Erstens: Das Beharren auf einem biomedizinischen Konzept ist aus wissenschaftlicher Sicht unhaltbar weder der Stamm eines entasteten Tannenbaums noch die abgehauenen Zweige sind lebendig. Zweitens: Aus Sicht der Praxis entstehen menschliche Katastrophen. Drittens hat niemand bewiesen, dass das biomedizinische Paradigma mit den Diagnoselisten zu Einsparungen führt.

Cicero fragte $63 \mathrm{v}$. Chr. vor dem römischen Senat: "Quo usque tandem abutere, Catilina, patientia nostra?» Auf heute übertragen, lässt sich dieses Zitat wie folgt anpassen: «Wie lange, ach wie lange noch, Politiker, Krankenkassenmanager und sogenannte Gesundheitsökonomen, missbraucht ihr unsere ärztliche Geduld?!»

\section{Literatur}

1 Thurston C et al. Psychosom Med. 2017;79:441-9.

2 Brühlmeier Rosenthal D. Soziales Elend nach Stopp oder Verweigerung von IV-Renten. Schweiz Ärztezeitung. 2017;98(24):785-7.

3 Mast H, Schneuwly F. Mehr Verständnis für die eidgenössische Versicherungsmedizin. Schweiz Ärztezeitung. 2017;98(28-29):918-21. 